\title{
L'aberration du budget global: le projet-pilote suisse le démontre
}

\section{Jürg Unger-Köppel}

Dr méd., membre du Comité central de la FMH, responsable du département Médecine et tarifs hospitaliers

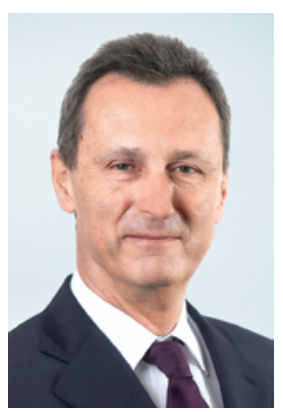

Lorsque les partenaires tarifaires n'arrivent pas à se mettre d'accord, la politique intervient. Alors que le budget global plane à l'horizon comme de gros nuages noirs, partisans et détracteurs se tournent vers l'Allemagne, le Canada et d'autres pays pour justifier leurs positions. Pourtant, il suffirait de prendre connaissance des résultats d'un projet-pilote mené en Suisse depuis 2004 pour voir l'impact d'un budget global ambulatoire sur le système de santé de notre pays.

Les institutions ambulatoires de psychiatrie de l'enfant et de l'adulte subissent un budget (de facto) global depuis l'introduction du TARMED en 2004, et ce pour la

\section{Avec un budget global, priorité aux urgences} et aux crises: les traitements à long terme sont proposés après de longs temps d'attente.

simple raison que le tarif purement au temps du TARMED génère des revenus qui ne couvrent que 40 à $80 \%$ des coûts et que le reste doit être payé par les cantons. Comme le tarif se réfère à un cabinet privé de 1997 sans informatique ni assistante médicale, il ne couvre pas les coûts informatiques, les hausse salariales, les tâches administratives et la gestion du cabinet. Par ailleurs, les heures de travail des collaborateurs sont fixées par contrat à la semaine. Le nombre d'heures pouvant être mis à la disposition des patients est par conséquent déterminé par la somme des taux horaires des collaborateurs ayant une activité clinique. Comme les coûts des collaborateurs ne sont pas couverts par les revenus du TARMED, le complément nécessaire pour chaque heure travaillée est à la charge des cantons, la plupart du temps sous la forme de prestations d'intérêt général (PIG). Comme les cantons ont tendance à les limiter, l'offre des institutions psychiatriques ambulatoires ne peut pas s'adapter aux besoins des patients. Pire encore, ces institutions sont obligées de se gérer au mieux avec un budget de facto global. Elles doivent tout d'abord prendre en charge les cas urgents et les patients en crise. Les traitements à long terme, susceptibles de réussir (le taux moyen de réus- site d'un traitement psychiatrique est de 70\%) ne sont proposés que de manière beaucoup trop insuffisante ou seulement après de longs temps d'attente. Les collaborateurs se sentent désemparés parce qu'ils voient les problèmes de leurs patients mais ne disposent pas de suffisamment de temps pour offrir de l'aide. Et les patients, enfants comme adultes, qui auraient de bonnes chances de guérir ou du moins de soulager leurs douleurs psychiques restent sans traitement ou avec un traitement insuffisant car les cabinets de psychiatrie n’arrivent pas à suivre.

Tout le monde prône «l'ambulatoire avant l'hospitalier». Or, en psychiatrie, les cliniques de jour considérées conformément à la LAMal comme des institutions ambulatoires se situent à mi-chemin entre l'ambulatoire et l'hospitalier. Elles permettent de raccourcir ou d'éviter des traitements hospitaliers chers mais sont aussi tributaires des prestations d'intérêt général pour couvrir leur frais. Comme les cantons diminuent ces dernières, les premières cliniques de jour ont dû fermer leurs portes, intensifiant de nouveau les hospitalisations. Une formule qui coûte cher et n'améliore pas le pronostic à long terme des patients concernés.

\section{Le budget de facto global pour les institutions psychiatriques ambulatoires est un échec.}

A l'instar du projet-pilote mené en Suisse depuis 2004 avec les psychiatres, le budget (de facto) global des institutions psychiatriques ambulatoires est un échec, une idée qui n'est rien d'autre qu'une aberration.

Aujourd'hui, le budget global ambulatoire figure au calendrier politique. Pour les tarifs, il importe que les partenaires tarifaires continuent de rechercher ensemble des solutions plutôt que de se livrer à une guerre de tranchées en tant qu'«adversaires tarifaires». C'est la seule voie pour éviter le budget global, cet instrument inapproprié pour les domaines de la prise en charge hospitalière et ambulatoire. Ce serait primordial pour les patients. 